\title{
25 Research Soure \\ Quantitative Microbial Population Study Reveals Geographical Differences In Bacterial Symbionts of Ixodes Ricinus
}

\author{
Aleksandra I. Krawczyk \\ RIVM: Rijksinstituut voor Volksgezondheid en Milieu
}

\section{Lisa Röttjers}

KU Leuven Rega Institute for Medical Research.: Katholieke Universiteit Leuven Rega Institute for Medical Research Manoj Fonville

RIVM: Rijksinstituut voor Volksgezondheid en Milieu

\section{Katsuhisa Takumi}

RIVM: Rijksinstituut voor Volksgezondheid en Milieu

\section{Willem Takken}

Wageningen University \& Research

\section{Karoline Faust}

KU Leuven Rega Institute for Medical Research: Katholieke Universiteit Leuven Rega Institute for Medical Research Hein Sprong ( $\nabla$ hsprong@gmail.com )

Rijksinstituut voor Volksgezondheid en Milieu https://orcid.org/0000-0002-0218-4320

\section{Research}

Keywords: Tick-borne disease, Spotted fever Rickettsiosis, transmission dynamics, quantitative microbiome analysis, low-biomass samples

Posted Date: July 27th, 2021

DOI: https://doi.org/10.21203/rs.3.rs-715041/v1

License: (c) (i) This work is licensed under a Creative Commons Attribution 4.0 International License. Read Full License 


\section{Abstract}

Background: Ixodes ricinus ticks vector pathogens that cause serious health concerns. Like in other arthropods, the microbiome may affect the tick's biology with consequences for pathogen transmission. Here, we explored the bacterial communities of $I$. ricinus across its developmental stages and six geographic locations by the 16S rRNA amplicon sequencing, combined with quantification of the bacterial load.

Results: A wide range of bacterial loads was found. Accurate quantification of low microbial biomass samples permitted comparisons to high biomass samples, despite the presence of contaminating DNA. The bacterial communities of ticks were associated with geographical location rather than life stage, and differences in Rickettsia abundance determined this association. Subsequently, we explored the geographical distribution of four verticallytransmitted symbionts identified in the microbiome analysis. For that, we screened 16,555 nymphs from 19 forest sites for Rickettsia helvetica, Rickettsiella spp., Midichloria mitochondrii, and Spiroplasma ixodetis. The infection rates of all vertically-transmitted symbionts differed between the study sites, and none of them was present in all tested ticks, suggesting facultative associations with I. ricinus. The proportions in which symbionts occurred in populations of I. ricinus were highly variable, but geographically close study sites expressed similar proportions. These patterns were in contrast to what we observed for the horizontally-transmitted pathogens Borrelia burgdorferi sensu lato, Anaplasma phagocytophilum, and Neoehrlichia mikurensis. Lastly, nearly $12 \%$ of tested nymphs were free of any targeted microorganisms, which is in line with the microbiome analyses.

Conclusions: Our results show that the microbiome of $I$. ricinus is highly variable, but changes gradually and ticks originating from geographically close forest sites express similar bacterial communities. This suggests that geography-related factors affect the infection rates of vertically-transmitted symbionts in I. ricinus. Since some symbionts, such as $R$. helvetica can cause disease in humans, we propose that public health investigations consider geographical differences in its infection rates.

\section{Introduction}

In Europe, Ixodes ricinus transmits a plethora of pathogens to humans, posing severe health concerns (1-3). Recently, there is an increasing interest in how the community of microorganisms inhabiting a tick, or the so-called microbiome, affects its fitness and vectorial capacity. In the long-term, understanding such effects of these microorganisms can lead to the development of novel and sustainable ways to combat ticks and tick-borne diseases, as it has been demonstrated for other arthropods $(4,5)$. The microbiome may influence tick-borne pathogen transmission dynamics, for instance, by inducing the host immune system or altering the integrity of the host gut layer (6). For example, studies have shown that the gut microbiota of the black-legged tick, l. scapularis, modulates the integrity of the peritrophic matrix that separates the lumen from the digestive cells of the gut and subsequently affects the establishment of Borrelia pathogens (7).

There is little known about how microorganisms, other than pathogenic agents, are acquired and propagated in $I$. ricinus ticks. Previous studies on the microbiome of I. ricinus detected large bacterial diversities, an overall unstable microbial composition, and an extremely low bacterial load in the midgut (8-12). The microbiome of Ixodes ticks has been shown to vary on a spatial scale $(13,14)$ and through the ontogeny $(13,15-17)$. Since $I$. ricinus has a three-host life cycle, feeding once per life stage (larva, nymph, and adult female but not male) on various vertebrates, each feeding is an opportunity for a tick to acquire microorganisms in addition to acquisition from the vegetation, where it spends most of its life (18). 
Various bacterial microorganisms such as Rickettsia helvetica, Spiroplasma ixodetis, Midichloria mitochondrii, and Rickettsiella spp. have evolved symbiotic relationships with I. ricinus $(13,19)$. These symbionts are predominantly transmitted vertically, namely, from female ticks to offspring $(20,21)$. Presumably, $R$. helvetica, S. ixodetis, M. mitochondrii, and Rickettsiella spp. are dependent on ticks for their survival in nature, impact their tick hosts, and some of them, e.g. R. Helvetica are potentially pathogenic to humans. However, since their roles in the biology and ecology of $I$. ricinus are not understood, it is unclear what factors determine their prevalences in tick populations. Contrary to vertically-transmitted symbionts, tick-borne microorganisms that are pathogenic to humans are predominantly horizontally-transmitted; through tick bites, via vertebrate hosts. These include pathogens such as Borrelia burgdorferi sensu lato, Anaplasma phagocytophilum, and Neoehrlichia mikurensis. Their infection prevalences in I. ricinus are mainly determined by local vertebrate communities $(22,23)$. Although some human tickborne pathogens are transmitted both horizontally and vertically (e.g. B. miyamotoi, Babesia divergens), the vertically-transmitted microorganisms from this study are referred to as symbionts, whereas the strictly horizontallytransmitted microbes as (human) pathogens.

In this study, we aimed to elucidate the dynamics of the bacterial community of I. ricinus with emphasis on symbionts, which may potentially influence tick vectorial capacity of pathogens. For that, we first used the 16s rRNA amplicon sequencing to explore the microbiome of I. ricinus ticks from six distinct geographical locations and of all life stages. Studies investigating the complete microbiome of Ixodes ticks often do not take a total bacterial load into account $(13,24-26)$. This can lead to spurious conclusions on the tick microbiome as low bacterial biomass might have important biological implications that are overlooked. Since the quantification of bacterial density can help better understand of tick physiology, this study adopted a qPCR approach inspired by studies on the accurate quantification of the bacterial microbiome $(27,28)$.

The microbiome analysis presented here allowed us to gain insight into microbial communities of $I$. ricinus and form the hypothesis that the prevalence of some vertically-transmitted tick symbionts is determined on a larger geographical scale than observed for horizontally-transmitted pathogens. To test this hypothesis, we screened with the symbiont-targeted qPCR nearly 17,000 individual questing nymphs of $I$. ricinus, collected from 19 forest sites in the Netherlands. Lastly, we compared distribution patterns of vertically-transmitted symbionts with horizontallytransmitted pathogen communities, which were quantified in a previous study (29).

\section{Materials And Methods}

Four different datasets were utilized in this study. Therefore, for clarity, each dataset has been summarized in Table 1.

Table 1. All datasets used in this study. 


\begin{tabular}{|c|c|c|c|c|c|c|}
\hline $\begin{array}{l}\text { Dataset } \\
\text { (name) }\end{array}$ & $\begin{array}{l}\text { Ticks } \\
\text { (n) }\end{array}$ & $\begin{array}{l}\text { Life } \\
\text { stages }\end{array}$ & $\begin{array}{l}\text { Locations in } \\
\text { the } \\
\text { Netherlands }\end{array}$ & $\begin{array}{l}\text { Year of } \\
\text { sampling }\end{array}$ & Molecular technique & Source \\
\hline Microbiome & $\begin{array}{l}655 \text { in } \\
133 \\
\text { pools }\end{array}$ & $\begin{array}{l}\text { Larvae, } \\
\text { nymphs, } \\
\text { adult } \\
\text { females } \\
\text { \& males }\end{array}$ & $\begin{array}{l}\text { AW, DK, ST, } \\
B U, H D, Z M \\
(n=6)\end{array}$ & 2016 & 16S rRNA sequencing & $\begin{array}{l}\text { This } \\
\text { study }\end{array}$ \\
\hline Symbiont & 16,555 & Nymphs & $\begin{array}{l}\text { AW, DK, ST, } \\
\text { BU, HD, ZM, } \\
\text { PD, SD, VH, } \\
\text { VA, MH, HM, } \\
\text { BB, PW, DW, } \\
\text { EN, RB, VL, } \\
\text { KB }(n=19)\end{array}$ & $\begin{array}{l}2013- \\
2014\end{array}$ & $\begin{array}{l}\text { qPCR targeting: } R \text {. helvetica, } S \text {. } \\
\text { ixodetis, } M \text {. } \\
\text { mitochondrii, Rickettsiella spp. }\end{array}$ & $\begin{array}{l}\text { This } \\
\text { study }\end{array}$ \\
\hline Pathogen & 13,967 & Nymphs & $\begin{array}{l}\text { AW, DK, ST, } \\
\text { BU, HD, ZM, } \\
\text { PD, SD, VH, } \\
\text { VA, MH, HM, } \\
\text { BB, PW, DW, } \\
\text { EN, RB, VL, } \\
\text { KB }(n=19)\end{array}$ & $\begin{array}{l}2013- \\
2014\end{array}$ & $\begin{array}{l}\text { qPCR targeting: } B \text {. } \\
\text { burgdorferi s.l., } A \text {. } \\
\text { phagocytophilum, N. mikurensis }\end{array}$ & $\begin{array}{l}\text { Takumi } \\
\text { et al., } \\
2019\end{array}$ \\
\hline $\begin{array}{l}\text { Transmission } \\
\text { mode }\end{array}$ & 1,130 & $\begin{array}{l}\text { Larvae, } \\
\text { nymphs, } \\
\text { adult } \\
\text { females } \\
\text { \& males }\end{array}$ & $\begin{array}{l}\text { AW, ST } \\
(n=2)\end{array}$ & 2019 & $\begin{array}{l}\text { qPCR targeting: R. helvetica, S. } \\
\text { ixodetis, M. } \\
\text { mitochondrii, Rickettsiella spp. B. } \\
\text { burgdorferi s.l., A. } \\
\text { phagocytophilum, N. mikurensis }\end{array}$ & $\begin{array}{l}\text { This } \\
\text { study }\end{array}$ \\
\hline
\end{tabular}

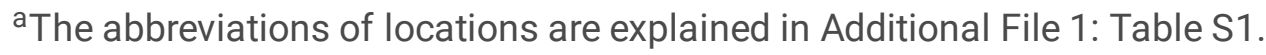

\section{Microbiome dataset}

\section{Sample collection and preparation for microbiome analyses of I. ricinus}

We utilized pools of I. ricinus larvae, nymphs, and individual adult females and males from six locations across the Netherlands for microbiome profiling (Table 1; Fig. 1, triangles). These locations were selected based on pre-existing knowledge of $B$. burgdorferi s.I. prevalence, the density of ticks, vegetation profile, and vertebrate community obtained in a cross-sectional study (22). The full names of the sites, their coordinates, and vegetation descriptions are provided in Additional file 1: Table S1.

Pooled and individual ticks from six forest sites (triangles) were used for a 16s rRNA amplicon sequencing analysis. Individual nymphs from these and 13 (points) other forest sites were tested by qPCR for the presence of tick symbionts. A box marks the sampling site by two letters, and a linear colour gradient represents latitude. Full coordinates, habitat, vegetation cover, tick densities, and a number of vertebrate species per locations are provided in Additional file 1: Table S1.

Questing I. ricinus were collected in 2016 by blanket dragging. In total, 655 ticks were combined into pools by life stage and location. All ticks were washed three times in 70\% ethanol, and DNA from 32 pools of 10 larvae, 18 pools of 5 nymphs, 18 pools of 10 nymphs, 34 individual female, and 31 individual male ticks was extracted using the 
Qiagen DNeasy Blood \& Tissue Kit according to the manufacturer's protocol (Qiagen, Venlo, The Netherlands). A sampling scheme and sample metadata are provided in Additional file 1: Table S2 and Table S3.

\section{Microbial profiling and taxonomic clustering}

Illumina MiSeq V3-V4 region of 16S rRNA amplicon libraries were generated and sequenced by BaseClear (Leiden, the Netherlands). The description of the method has been published previously (30). Sequenced reads were imported to CLC Genomics Workbench 10.0.1 supplemented with CLC Microbial Genomics Module 3.6.1 (www.clcbio.com). Overlapping pairs of raw reads were merged into single longer reads and trimmed with a quality score limit of 0.05 and 2 ambiguous nucleotides. At this stage, primer sequences were trimmed. Subsequently, reads were fixed-length trimmed ( $400 \mathrm{bp}$ ). To identify operational taxonomic units (OTUs), reads were clustered using the reference databases SILVA 16S version 128 with 97\% identity as the clustering criterion; chimeras were removed.

\section{S rRNA quantification and total bacterial load}

Total bacterial load in all samples was quantified, and proportions were multiplied by the load to convert relative into absolute abundances. Quantification of total bacterial DNA load was determined by 16S rRNA qPCR as previously described (31). The total bacterial load is a cost-effective and scalable solution for datasets of this size, since quantification methods through flow cytometry are not compatible with the sampling technique. Samples were normalized to control for arbitrary variation in sequencing depth, and the normalized abundances were scaled by the 16S rRNA qPCR values of each sample. For diversity analyses, samples were rarefied to the lowest sequencing depth.

\section{Microbiome analyses}

All analyses were carried out in R 3.6.0 (32). We used the R package vegan (version 2.5-6) for ordinations, diversity indices, and fitting of environmental vectors or factors onto ordinations (33). We also computed silhouette scores from the Bray-Curtis dissimilarities and Sheldon evenness (34). All principal coordinate analyses were carried out using Bray-Curtis dissimilarities, and envfit correlation to the principal components was corrected for multipletesting with the Benjamini-Hochberg correction. Additionally, we carried out PERMANOVA with the adonis function from $\mathrm{R}$ package vegan to assess whether tick life stage significantly affected community variation. We tested for multivariate spread through the betadisperfunction.

To test how well different factors explained clusters observed on the PCoA plots, we calculated the Bray-Curtis dissimilarities and evaluated cluster quality as the silhouette score with the factors as cluster labels. The silhouette score, bounded by -1 and 1, takes both cluster cohesion and cluster separation into account. We used k-means clustering of the log-transformed Rickettsia abundances to compute silhouette scores for Rickettsia.

To investigate correlations between OTUs and tick life stage, we fitted proportional odds models with OTU abundances scaled by the total bacterial load as the dependent variable and used tick life stage as independent variables. Details on models are provided in Additional file 2: Text S1. We compared these models with the likelihood ratio test, using an implementation of Nagelkerke's pseudo- $\mathrm{R}^{2}$ from the $\mathrm{R}$ package rcompanion (version 2.3.7) (35).

\section{Transmission mode dataset}


A total of 1,130 I. ricinus ticks of all developmental stages were collected in 2019 from two locations (ST and AW) and tested individually with qPCR for the presence of $S$. ixodetis, R. helvetica, B. burgdorferi s.l., A. phagocytophilum, and $N$. mikurensis for which primers and probes have been developed and published before (36-39). In addition, ticks were tested for Rickettsiella spp., M. mitochondrii for which primers and probes were designed in this study. All targeted genes and qPCR protocol are provided in Additional file 2: Text S2. Subsequently, pathogens and symbionts were assigned the transmission mode based on their presence or absence in the larval stage indicating vertical or horizontal transmission, respectively.

\section{Dataset on vertically-transmitted symbionts (Symbiont dataset)}

To determine the geographic distribution and prevalence of tick symbionts, we analysed a total of 16,555 ticks, which were collected in a previous cross-sectional study $(22,40)$. Briefly, questing nymphs of $I$. ricinus were collected from 19 locations in forested areas in the Netherlands in 2013 and 2014 (Fig. 1, triangles and dots). Details on data collection were described previously $(22,40)$. We tested questing individual nymphs of $I$. ricinus for the presence of $S$. ixodetis, R. helvetica, Rickettsiella spp., and M. mitochondrii.

\section{Dataset on horizontally-transmitted pathogens (Pathogen dataset)}

In addition, we included in our analysis data on the prevalence and distribution of tick-borne pathogens (Table 1). This data was generated from the same tick collection, from 13,967 of 16,555 ticks, in the study of Takumi et al. (29). The pathogens included A. phagocytophilum, N. mikurensis, B. miyamotoi, and three genospecies of $B$. burgdorferi s.l. as follows: $B$. afzelii, $B$. garinii, and B. valaisiana. Data on B. garinii and $B$. valaisiana were combined for further analysis as they are both considered bird-borne pathogens.

\section{Relative occurrence of vertically-transmitted tick symbionts and horizontally-transmitted pathogens}

Based on the presence and absence of microorganisms detected with qPCR (Symbiont and Pathogen datasets), we assigned each tick a haplotype, an integer between 0 and $2^{n-1}$. Each integer corresponds to one of $2^{n}$ distinct outcomes for a series of $\mathrm{n}$ qPCR tests performed on the tick. Haplotype frequencies were arranged in a table where rows are the sampling sites and columns are the observed haplotypes. Each row was divided by the row sum. The column mean was subtracted from each column. Principal component analysis of the data table was performed by applying the singular value decomposition (41).

\section{Results}

\section{Microbiome dataset}

\section{Microbial profiling and taxonomic clustering}

A total of 131 of 133 processed samples generated 18,803,386 raw reads on Illumina MiSeq flow cell. Two samples failed at the amplification stage, probably due to low bacterial DNA load. A total of $6,013,524$ sequences were 
assigned taxonomy. A total of 4,978 unique OTUs were identified (Additional file 3: Table S4), and the top 10 most abundant taxa consisted of Rickettsia, Rickettsiella, Midichloria, Pseudomonas, Halomonas, Rickettsiella, Mycobacterium, Shewanella, Methylobacterium, and Williamsia.

Three taxa, Pseudomonas, Halomonas, and Shewanella, were the most abundant in negative controls, indicating that these microorganisms are contamination from the processing or sequencing pipeline. All counts belonging to these taxa were binned into a synthetic 'Contaminant' taxon (Fig. 2). Several tick samples had extremely low biomass, and their community composition was similar to that of sequenced negative controls (Additional file 3 : Figure S1). These samples lacked tick-associated symbionts, and since the absence of a high-abundance symbiont can be considered a biological phenomenon, we chose to retain these samples while the negative controls were excluded from further analysis. A full list of OTUs identified in the negative controls is provided in Additional file 3: Table S4.

Samples are separated by quartiles of total bacterial load (16S rRNA content in $\mathrm{ng} / \mu \mathrm{L}$ ). Bacteria present at a minimum threshold in sequenced control samples (blanks) were binned into the artificial 'Contaminant' phylum. Taxa not in the top 10 were labelled 'Other'. A full list of detected bacterial taxa is provided in Additional file 3: Table S4.

Rarefaction curves indicated sufficient sequencing coverage for most samples, as demonstrated by most observed OTU accumulation curves having reached a plateau at 2000 reads (Additional file 3: Figure S2). The low amount of total DNA and pooling strategy resulted in highly uneven sequencing depths Additional file 3: Figure S3a). We assessed whether sequencing depth correlated to diversity since this would necessitate a rarefaction step. There was no apparent correlation between sequencing depth and diversity (Additional file 3: Figure S4a), linear regression model $\left.p=0.907, R^{2}=-0.008\right)$, and we chose to avoid rarefaction before additional analyses were carried out.

\section{Abundance of tick-associated symbionts}

Known members of the tick microbiome $(19,42,43)$, the genera Rickettsia, Midichloria, and Rickettsiella were among 10 of the most abundant taxa in the overall microbiome dataset accounting for $24.4 \%, 6.3 \%$ and $17.1 \%$ of all reads, respectively (Fig. 2). Another tick-associated microorganism, Spiroplasma, was abundant but only in samples from two locations, ZM and AW, accounting for $0.3 \%$ of all reads (44). The most abundant tick-borne pathogen in the dataset was Neoehrlichia (0.3\%). Other pathogenic genera, including Borrelia and Anaplasma, constituted a small part of the overall tick microbiome accounting for $0.1 \%$ and $0.02 \%$ of all reads, respectively. Lastly, $0.4 \%$ of reads represented Wolbachia genus, probably due to endoparasitoid Ixodiphagus hookeri eggs in ticks (TijsseKlasen et al., 2011; Plantard et al., 2012).

\section{Effect of total bacterial load on the abundance of contaminants}

Total bacterial load was weakly but significantly correlated with sequencing depth (Additional file 3: Figure S5a, linear regression model $p=0.011, R^{2}=0.042$ ). Moreover, $89.3 \%$ of samples had lower total bacterial load than the mean total bacterial load, meaning that the bacterial load was heterogeneous and skewed towards lower loads. Bacterial load was strongly correlated with the total number of uncorrected counts belonging to Pseudomonas, Shewanella, or Halomonas (Additional file 3: Figure S5b, linear regression model $p<0.001, R^{2}=0.608$ ). After scaling normalized sequencing counts by the total bacterial load, the bar plots of bacterial abundances demonstrate that the contaminants make up a large fraction of samples with low total bacterial loads (Fig. 2 and Additional file 3: Figure S3b). In addition, the lower total bacterial load was correlated to the higher Shannon diversity (Additional file 3: Figure S4b, linear regression model, $\left.p<0.001, R^{2}=0.195\right)$. 


\section{Effect of life stage and longitude on bacterial community variation}

The marginal effect of life stage on bacterial community variation was significant, but the $R^{2}$ was $s m a l l(p=0.001$, $\mathrm{R}^{2}=0.121$, Additional file 4: Table S5). Our test for multivariate homogeneity of group dispersion was highly significant ( $<<0.001$, Additional file 4: Table S5). The silhouette score from the Bray-Curtis dissimilarities was 0.024 , suggesting that samples are not tightly grouped by life stage (Fig. 3).

Since the response of the bacterial community to tick life stage might not necessarily reflect the response of specific taxa, we investigated the link between Rickettsia, Rickettsiella and life stage in more detail. Both Rickettsia and Rickettsiella had significant correlations to the axes ( $p=0.001$ for each), with a silhouette score of 0.122 for Rickettsia-based clusters suggesting that it could better explain community structure compared to tick life stage (Fig. 4). However, proportional odds models suggest that high Rickettsia and Rickettsiella abundances could not be explained with a model including only life stage as a factor (Additional file 4: Table S6). For both Rickettsia and Rickettsiella, the pseudo- $\mathrm{R}^{2}$ was low at 0.051 and 0.009 respectively.

Principal Coordinate Analysis of Bray-Curtis dissimilarities overlaid with envfit vectors for tick symbionts. Of these abundance vectors, only Rickettsia and Rickettsiella correlated significantly to the principal components ( $p=0.001$ for both). Nagelkerke's pseudo- $\mathrm{R}^{2}$ for different ordinal logistic regression models fitting scaled Rickettsia and Rickettsiella abundances is provided in Additional file 4: Table S6.

\section{Transmission mode dataset}

Rickettsia helvetica, S. ixodetis, Rickettsiella spp., and M. mitochondrii were detected in all life stages (Additional file 4: Table S7), indicating vertical transmission. Borrelia burgdorferi s.I. (except one larva), A. phagocytophilum, and N. mikurensis were only detected in nymphal and adult stages (Additional file 4: Table S7). Our results corroborate the latter symbionts enter a tick population via horizontal transmission while larvae feed on an infected host (45-47).

\section{Symbiont and Pathogen datasets Prevalence of tick symbionts and pathogens}

The vertically-transmitted symbionts were detected to a varying degree in all 19 forest sites (Fig. 5). Data on prevalence per symbiont per forest site as well as data generated in the previous study on three $B$. burgdorferis.I. genospecies including B. afzelii, B. garinii and B. valaisiana (combined), A. phagocytophilum, and N. mikurensis are provided in Additional file 4: Table S8. None of the nine symbionts were detected in $12 \%$ of the tested nymphs $(n=$ $1,668)$, agreeing with the $16 \mathrm{~S}$ rRNA amplicon sequencing results where many samples contained almost no microbiome (Additional file 3: Figure S1).

\section{Relative occurrence of vertically-transmitted symbionts}

We delineated the forest sites into three clusters according to specific combinations of infections in individual ticks (Fig. 1 and Fig. 6a). In cluster one, we found abundant questing nymphs in which all symbionts were absent (h00; Table S9 lists all the haplotypes) or in which only M. mitochondrii (h08 haplotype) was detected. In cluster two, questing nymphs were abundant, in which Rickettsiella spp. was present together with M. mitochondrii (h12) or 
without this species (h04). In cluster one, we found questing nymphs with $R$. helvetica and another symbiont: $M$. mitochondrii, Rickettsiella spp., and R. helvetica (h13), Rickettsiella spp. and R. helvetica (h05), or M. mitochondrii and $R$. helvetica (h09). These three haplotypes (h13, h05, and h09) lacked S. ixodetis.

\section{Relative occurrence of horizontally-transmitted pathogens}

To contrast these results against pathogens that are transmitted horizontally, we performed the same analysis on $A$. phagocytophilum, N. mikurensis, B. afzelii, B. garinii and B. valaisiana (combined). Contrary to the verticallytransmitted symbionts, the forest sites did not cluster according to geographic proximity (Fig. 1 and Fig. 6b). The principal driver for differentiating the sampling sites was the absence of any of the horizontally-transmitted pathogens in questing nymphal ticks (h00).

\section{Discussion}

\section{Variable bacterial loads}

Analysing ticks with next-generation sequencing comes with methodological challenges related to their overall low biomass. Samples with low total bacterial load suffer relatively more from contaminant DNA and crosscontamination than samples with high bacterial load (48). Therefore, pooling tick samples can maximize microbial yield and provide a representative sample of microbial taxonomic diversity $(13,49,50)$. We used a similar approach in our study. Although we expected that the pooling would obfuscate the effects of bacterial biomass on community structure, 16S rRNA quantification demonstrated a surprisingly wide range of total bacterial loads across pools of ticks analysed in our experiment. While we could not determine the total bacterial load for a single tick directly, the negative correlation between established contaminants and total bacterial load suggests that our low microbial biomass pooled samples were more affected by contaminating DNA than high biomass samples (Additional file 3: Figure S5b). A similar negative correlation was found for Shannon diversity and total bacterial load, suggesting that diversity metrics are inflated for these low-biomass pools (Additional file 3: Figure S4b), possibly due to higher sampling depth (51). However, by scaling microbial abundances through $16 \mathrm{~S}$ qPCR quantification, the absolute abundances of the contaminants and rare species were reduced while absolute abundances of high-density samples were reflective of the community. Our results show that quantitative techniques for microbiome studies can accurately identify low-biomass samples in data sets with heterogeneous microbial load, even when used in combination with a pooling strategy commonly used to sequence low-biomass arthropod microbiomes.

\section{Limited microbiome}

We observed similarities between sequenced negative controls and low-biomass samples. Many I. ricinus individuals appeared to harbour a limited microbiome, and whatever microbiome they did harbour, could not be distinguished from negative controls based on Bray-Curtis dissimilarity (Additional file 3: Figure S1). However, since this dissimilarity takes absolute counts into account, it emphasizes the low biomass of these samples rather than their composition. The genera found in these samples did appear to be distinct, with low-biomass samples containing genera unique to the tick microbiome such as Midichloria, Rickettsia, Rickettsiella, and Spiroplasma (Additional file 3: Figure S6). Therefore, we chose to retain these samples since they may represent a condition of biological interest: the absence of any abundant tick symbiont. Yet, some samples had low relative abundance or were devoid of these genera, which are vertically-transmitted symbionts previously associated with I. ricinus (52). We analysed a similar tick population with symbiont targeted qPCR, to test this hypothesis since next generation sequencing approaches cannot distinguish true absence from absence due to low sequencing depth. Interestingly, 
$12 \%$ of $~ 15,000$ questing nymphs did not carry any of the four above-mentioned vertically-transmitted symbionts and any of five horizontally-transmitted pathogens (B. afzelii, B. garinii, B. valaisiana, A. phagocytophilum, or N. mikurensis). In addition, with the microbiome analysis, we did not identify any other potential symbiont of $I$. ricinus.

Our results suggest that individual ticks can live devoid of bacterial symbionts. However, it remains unclear whether they can successfully feed or reproduce and, if not, what their role is in sustaining a tick population. Alternatively, ticks can harbour undetectable, by techniques used in this study, abundances/loads of bacterial symbionts. This could be happening when ticks are not actively metabolizing. Possibly, to maintain a symbiotic relationship, ticks downregulate the growth of intracellular symbionts during periods when symbiosis is the most expensive. For instance, in tsetse flies and algae, the load of symbionts has been observed to change through host development and environmental perturbations (such as changing humidity and light levels) $(53,54)$. Lastly, I. ricinus might possess viral, eukaryotic or perhaps archaea bacteria as obligatory symbionts.

We propose that $I$. ricinus as a species possess a highly variable microbiome and no obligate bacterial symbiont present in $100 \%$ of the population. These indications are somewhat surprising given that ticks are arthropods feeding exclusively on vertebrate blood, a nutritionally restricted diet. In many hematophagous arthropods, including other tick species, obligate and maternally acquired bacterial symbionts are necessary for providing essential metabolites such as vitamins $B$ that are deficient in vertebrate blood (55). It is also possible that multiple symbionts can fulfil this and other crucial functions in I. ricinus, so that ticks may only need a single facultative symbiont from a pool of candidates. For instance, in aphids, Serratia symbiotica and Hamiltonella defensa confer similar benefits to their hosts. Both symbionts have been shown to defend aphids against a parasitoid wasp (56) and improve host survival when subjected to heat shock (57). Here, we observed that $S$. ixodetis occurred at the highest prevalence in ticks from the study sites with the lowest prevalence of $R$. helvetica (Fig. 5). Possibly, these two symbionts play similar biological function in $I$. ricinus and bearing both of them is energetically costly to ticks.

When it comes to the lack of an obligate symbionts, similar observations have been made for I. scapularis. Previously $R$. buchneri was considered an obligate symbiont of this tick species as its genome contains all the genes of de novo folate (vitamin B9) biosynthesis (58). However, on many occasions, l. scapularis ticks without $R$. buchneri have been reported. The prevalence of this symbiont in tick populations varied between 46-82\% depending on the location, suggesting a facultative over the obligate relationship $(59,60)$.

In addition, an in-depth study on I. scapularis suggested an unstable midgut microbiome (61), and a recent study by Guizzo et al. (12) showed an extremely low overall bacterial load in the I. ricinus midgut. These results are in line with an increasing body of evidence that many animals, including arthropods, are minimally or facultatively dependent on bacterial microbes or may not need a microbiome at all (62). Thus, the observed variability of the tick microbiome likely represents transient associations with bacteria from the abiotic and biotic environment. In the recent review by Narasimhan et al. (63), it has been discussed that these transient microbial associations are under surveillance by tick innate immune responses. Subsequently, many microbes are cleared by effector molecules such as antibacterial peptides and potentially excreted due to the absence of cognate adhesins to engage with the tick gut (63).

\section{Microbiome of different tick life stages}

We have observed small but statistically significant differences between microbiome compositions of distinct developmental stages of I. ricinus. The developmental stage on its own did not explain the clustering of tick microbiomes (Fig. 3A). Contrary to what we had expected, bacterial diversity seems to decrease along with tick 
development, illustrated with a significant difference between larvae and females. However, the opposite trend was observed from nymphs to adult males. Furthermore, the results suggest that during the off-host phases of ticks, fewer bacterial species enter a tick than has been speculated before (8). Alternatively, the decreased bacterial diversity in females that we observed arose from the technical limitations of next-generation sequencing. It has been shown that abundant bacteria recruit more reads and potentially mask less abundant ones making them less likely to be detected (64).

Previous studies have reported significant differences in Ixodes microbiome compositions of distinct life stages. However, they reported contradictory results regarding the dynamics of bacterial diversity. Zolnik et al. $(15,26)$ documented increasing diversity, while Kwan et al. (24), Swei \& Kwan (25), and Carpi et al. (13) showed decreasing diversity along with tick development. Therefore, the role of the life stage in shaping the tick microbiome is still unclear, and it is difficult to subtract this information from the data obtained solely with next-generation sequencing. Thus, in future studies, combining this method with other detection techniques is highly advisable.

\section{Microbiome and geographical location}

In this study, the microbiomes of $I$. ricinus differed between distinct geographical locations. By scaling locations by their longitude, we could visualize that the change in the tick microbiomes occurred gradually rather than randomly (Fig. 3B). Thus, the bacterial communities of ticks originating from geographically distinct forest sites were significantly different and clustered apart. Previous studies also showed that the microbiome of Ixodes ticks varies by geographic location $(13,14)$. These studies have given several probable explanations for differences in bacterial communities suggesting that they arose from the distinct habitats with a dissimilar availability of vertebrate hosts and consequently different environmental and animal host-associated bacteria (13). Nevertheless, both differences in habitat and animal composition are locally determined, and the results in our study indicate that the factors driving differences in the microbiome are distributed over a broader geographical range.

\section{Distribution of tick symbionts}

Yhe observed clustering of the microbiomes was predominantly caused by varying tick symbiont communities. The strongest determinants were the abundance and prevalence of Rickettsia and Rickettsiella (Fig. 4). Therefore, based on these results, we hypothesized that the distribution and prevalence of $R$. helvetica (the most common Rickettsia species in I. ricinus) and Rickettsiella spp. is determined on a larger spatial scale than a single forest site. In addition to these symbionts, we studied $S$. ixodetis and $M$. mitochondrii, which were highly abundant in our microbiome dataset but less responsible for variations in bacterial communities of ticks. The prevalences of $R$. helvetica, Rickettsiella spp., M. mitochondrii, and S. ixodetis in questing nymphs were significantly different between the forest sites. Although all symbionts were present in all 19 studied locations and often at high prevalence, they occurred in varying proportions. The forest sites, which expressed similar proportions of symbionts, were also geographically close, consistent with what we observed in the microbiome dataset (Fig. 3B and Fig. 6A). We did not observe this pattern in horizontally-transmitted pathogens such as B. afzelii, B. garinii, A. phagocytophilum, and N. mikurensis. The proportions in which they occurred were more random and varied between geographically close forest sites (Fig. 6B). This result was not surprising because horizontally-transmitted pathogens have been shown to be determined mainly by local vertebrate communities $(22,23)$.

Nevertheless, mechanisms causing the heterogenicity in the prevalence of tick symbionts remain to be determined. Previous studies on host-symbiont interactions correlated variation in symbiont prevalence with environmental variables: a symbiont is more prevalent in the tick host population when it provides tolerance to a given biotic or abiotic stress, and less prevalent when it is less beneficial for mitigating stress (and therefore, stress is a weaker 
selective force; $(19,65)$. Therefore, (meta)populations of ticks from the western, northern, and central regions of the Netherlands may be exposed to different stresses. Abiotic stresses may arise from differences in soil type, temperature, and humidity related to coastal (west) and inland (centre and north) areas of the Netherlands. Biotic stresses such as the presence and abundance of tick parasites, for example, parasitic wasps, might lead to varying prevalences of facultative defensive symbionts in tick populations as demonstrated for other species of arthropods (66). Alternatively, the varying prevalences of a symbiont between distinct tick (meta)populations may arise from differences in transmission rate. The transmission rate could be potentially affected by a tick or symbiont genotypes or the compatibility of particular tick - symbiont genotype pairs (65). This evolutionary divergence within I. ricinus and/or a symbiont species could be promoted by a geographical barrier and limited exchange of tick individuals between different parts of the country. However, this variation has not yet been studied and documented for tick - microbe symbiosis, and here, we did not look at the genetic diversity of neither I. ricinus nor symbionts.

\section{Conclusions}

Ixodes ricinus has a limited bacterial microbiome both in load and diversity and appears to lack an obligate symbiont. Nevertheless, given the relatively high prevalences of a variety of symbionts such as $S$. ixodetis, $R$. helvetica, M. mitochondrii, and Rickettsiella spp. in questing ticks, symbiotic bacteria appear to be intertwined with the biology of ticks. Varying symbiont functions and mechanisms underlying tick-symbiont interactions, which remain unidentified, may affect their distribution in tick populations. This, in turn, may have significant ramifications for generating the risk of diseases caused by $S$. ixodetis and $R$. helvetica. Here, we learned that $R$. helvetica, displays characteristic geographical differences being highly prevalent in the coastal area vs inland. It is striking that such significant variation in prevalence can be observed already in a (small) country like the Netherlands. Whether this phenomenon also holds true for other more severe Spotted Fever Group Rickettsia's from other tick species, like $R$. conorii and $R$. rickettsii, is uncharted territory and subject of future studies. Our findings imply that public health investigations and measures for etiological agents, such as risk assessments, implementation of diagnostic modalities, and preventive measures, should consider these geographical differences in the prevalence of $R$. helvetica.

\section{Declarations}

\section{Ethics approval and consent to participate}

Not applicable

\section{Consent for publication}

Not applicable

\section{Availability of data and material}

De-multiplexed raw sequence reads supporting the conclusions of this article will be deposited into the Sequence Read Archive (SRA, http://www.ncbi.nlm.nih.gov/sra). The rest of the data generated or analysed during this study are included in this published article and its additional files.

\section{Competing interests}

The authors declare that they have no competing interests. 


\section{Funding}

This study was financially supported by the Dutch Ministry of Health, Welfare and Sport (VWS), and by a grant from the European Interreg North Sea Region program, as part of the NorthTick project. The funders had no role in the study design and interpretation, or the decision to submit the work for publication.

\section{Authors' contributions}

AlK and HS conceived and designed the study. AIK, LR, KF, MF, and KT contributed to the data collection and analysis. AlK, HS, LR, KF, and WT contributed to the data interpretation and manuscript preparation. All authors read and approved the final manuscript.

\section{Acknowledgements}

We thank Mei Ling Chu (RIVM) for helping with the 16S rRNA quantification.

\section{References}

1. Heyman P, Cochez C, Hofhuis A, van der Giessen J, Sprong H, Porter SR, et al. A clear and present danger: tickborne diseases in Europe. Expert Rev Anti Infect Ther. 2010;8(1):33-50.

2. Randolph S, Šumilo D. Tick-borne encephalitis in Europe: dynamics of changing risk. In: Takken Wand Knols BGJ, editor. Emerging pests and vector-borne diseases in Europe. Wageningen Academic Publishers; 2007. pp. 187-206.

3. Sprong H, Azagi T, Hoornstra D, Nijhof AM, Knorr S, Baarsma ME, et al. Control of Lyme borreliosis and other Ixodes ricinus-borne diseases. Parasit Vectors. 2018;11(1):145.

4. Mancini MV, Spaccapelo R, Damiani C, Accoti A, Tallarita M, Petraglia E, et al. Paratransgenesis to control malaria vectors: a semi-field pilot study. Parasit Vectors. 2016;9(1):1-9.

5. Ricci I, Damiani C, Rossi P, Capone A, Scuppa P, Cappelli A, et al. Mosquito symbioses: from basic research to the paratransgenic control of mosquito-borne diseases. J Appl Entomol. 2011;135(7):487-93.

6. Gall CA, Reif KE, Scoles GA, Mason KL, Mousel M, Noh SM, et al. The bacterial microbiome of Dermacentor andersoni ticks influences pathogen susceptibility. ISME J. 2016;10(8):1846-55.

7. Narasimhan S, Rajeevan N, Liu L, Zhao YO, Heisig J, Pan J, et al. Gut microbiota of the tick vector Ixodes scapularis modulate colonization of the Lyme disease spirochete. Cell Host Microbe. 2014;15(1):58-71.

8. Narasimhan S, Fikrig E. Tick microbiome: the force within. Trends Parasitol. 2015;31(7):315-23.

9. Greay TL, Gofton AW, Paparini A, Ryan UM, Oskam CL, Irwin PJ. Recent insights into the tick microbiome gained through next-generation sequencing. Parasit Vectors. 2018;11(1):12.

10. Aivelo T, Norberg A, Tschirren B. Bacterial microbiota composition of Ixodes ricinus ticks: the role of environmental variation, tick characteristics and microbial interactions. PeerJ. 2019;7:e8217.

11. Aivelo T, Norberg A, Tschirren B. Human pathogen co-occurrence in Ixodes ricinus ticks: effects of landscape topography, climatic factors and microbiota interactions. bioRxiv. 2019:559245.

12. Guizzo MG, Neupane S, Kucera M, Perner J, Frantová H, da Silva Vaz IJ, et al. Poor unstable midgut microbiome of hard ticks contrasts with abundant and stable monospecific microbiome in ovaries. Front Cell Infect Mi. 2020;10:211. 
13. Carpi G, Cagnacci F, Wittekindt NE, Zhao F, Qi J, Tomsho LP, et al. Metagenomic profile of the bacterial communities associated with Ixodes ricinus ticks. PLoS One. 2011;6(10):e25604.

14. Van Treuren W, Ponnusamy L, Brinkerhoff RJ, Gonzalez A, Parobek CM, Juliano JJ, et al. Variation in the Microbiota of Ixodes Ticks with Regard to Geography, Species, and Sex. Appl Environ Microbiol. 2015;81(18):6200-9.

15. Zolnik CP, Prill RJ, Falco RC, Daniels TJ, Kolokotronis SO. Microbiome changes through ontogeny of a tick pathogen vector. Mol Ecol. 2016;25(19):4963-77.

16. Kwan JY, Griggs R, Chicana B, Miller C, Swei A. Vertical vs. horizontal transmission of the microbiome in a key disease vector, Ixodes pacificus. Mol Ecol. 2017;26(23):6578-89.

17. Swei A, Kwan JY. Tick microbiome and pathogen acquisition altered by host blood meal. ISME J. 2017;11(3):813-6.

18. Needham GR, Teel PD. Off-host physiological ecology of ixodid ticks. Annu Rev Entomol. 1991;36(1):659-81.

19. Duron O, Binetruy F, Noel V, Cremaschi J, McCoy KD, Arnathau C, et al. Evolutionary changes in symbiont community structure in ticks. Mol Ecol. 2017;26(11):2905-21.

20. Parola P, Beati L, Cambon M, Raoult D. First isolation of Rickettsia helvetica from Ixodes ricinus ticks in France. Eur J Clin Microbiol Infect Dis. 1998;17(2):95-100.

21. Tully JG, Rose DL, Yunker CE, Carle P, Bove JM, Williamson DL, et al. Spiroplasma ixodetis sp. nov., a new species from Ixodes pacificus ticks collected in Oregon. Int J Syst Evol Microbiol. 1995;45(1):23-8.

22. Takumi K, Sprong H, Hofmeester TR. Impact of vertebrate communities on Ixodes ricinus-borne disease risk in forest areas. Parasit Vectors. 2019;12(1):1-12.

23. Van Buskirk J, Ostfeld RS. Controlling Lyme disease by modifying the density and species composition of tick hosts. Ecol Appl. 1995;5(4):1133-40.

24. Kwan JY, Griggs R, Chicana B, Miller C, Swei A. Vertical vs. horizontal transmission of the microbiome in a key disease vector, Ixodes pacificus. Mol Ecol. 2017;26(23):6578-89.

25. Swei A, Kwan JY. Tick microbiome and pathogen acquisition altered by host blood meal. ISME J. 2017;11(3):813-6.

26. Zolnik CP, Prill RJ, Falco RC, Daniels TJ, Kolokotronis SO. Microbiome changes through ontogeny of a tick pathogen vector. Mol Ecol. 2016;25(19):4963-77.

27. Bogaert D, Keijser B, Huse S, Rossen J, Veenhoven R, Van Gils E, et al. Variability and diversity of nasopharyngeal microbiota in children: a metagenomic analysis. PloS one. 2011;6(2):e17035.

28. Jian C, Luukkonen P, Yki-Järvinen H, Salonen A, Korpela K. Quantitative PCR provides a simple and accessible method for quantitative microbiota profiling. PLoS One. 2020;15(1):e0227285.

29. Takumi K, Sprong H, Hofmeester TR. Impact of vertebrate communities on Ixodes ricinus-borne disease risk in forest areas. Parasit Vectors. 2019;12(1):1-12.

30. Gommers LM, Ederveen TH, van der Wijst J, Overmars-Bos C, Kortman GA, Boekhorst J, et al. Low gut microbiota diversity and dietary magnesium intake are associated with the development of PPI-induced hypomagnesemia. FASEB J. 2019;33(10):11235-46.

31. Bogaert D, Keijser B, Huse S, Rossen J, Veenhoven R, Van Gils E, et al. Variability and diversity of nasopharyngeal microbiota in children: a metagenomic analysis. PloS one. 2011;6(2):e17035.

32. (2019) RCT. R: A language and environment for statistical computing. R Foundation for Statistical Computing, Vienna, Austria. URL http://www.R-project.org/.

Page $14 / 22$ 
33. Oksanen J, Kindt R, Legendre P, O’Hara B, Stevens MHH, Oksanen MJ, et al. The vegan package. Community ecology package. 2007;10:631-7.

34. Sheldon AL. Equitability indices: dependence on the species count. Ecology. 1969;50(3):466-7.

35. Mangiafico S, Mangiafico MS. Package 'rcompanion'. Cran Repos. 2017:1-71.

36. Krawczyk Al, Van Duijvendijk GL, Swart A, Heylen D, Jaarsma RI, Jacobs FH, et al. Effect of rodent density on tick and tick-borne pathogen populations: consequences for infectious disease risk. Parasit Vectors. 2020;13(1):1-17.

37. Jahfari S, Fonville M, Hengeveld P, Reusken C, Scholte EJ, Takken W, et al. Prevalence of Neoehrlichia mikurensis in ticks and rodents from North-west Europe. Parasit Vectors. 2012;5(1):74.

38. Heylen D, Tijsse E, Fonville M, Matthysen E, Sprong H. Transmission dynamics of Borrelia burgdorferi s.I. in a bird tick community. Environ Microbiol. 2013;15(2):663-73.

39. Courtney JW, Kostelnik LM, Zeidner NS, Massung RF. Multiplex real-time PCR for detection of anaplasma phagocytophilum and Borrelia burgdorferi. J Clin Microbiol. 2004;42(7):3164-8.

40. Hofmeester TR, Sprong H, Jansen PA, Prins HHT, van Wieren SE. Deer presence rather than abundance determines the population density of the sheep tick, Ixodes ricinus, in Dutch forests. Parasit Vectors. 2017;10(1):433.

41. Golub GH, Van Loan CF. The singular value decomposition and unitary matrices. Matrix Comp. 1996:70-1.

42. Sassera D, Beninati T, Bandi C, Bouman EA, Sacchi L, Fabbi M, et al. 'Candidatus Midichloria mitochondrii', an endosymbiont of the tick Ixodes ricinus with a unique intramitochondrial lifestyle. Int J Syst Evol Microbiol. 2006;56(Pt 11):2535-40.

43. Sprong H, Wielinga PR, Fonville M, Reusken $\mathrm{C}$, Brandenburg AH, Borgsteede $F$, et al. Ixodes ricinus ticks are reservoir hosts for Rickettsia helvetica and potentially carry flea-borne Rickettsia species. Parasit Vectors. 2009;2(1):41.

44. Henning K, Greiner-Fischer S, Hotzel H, Ebsen M, Theegarten D. Isolation of Spiroplasma sp. from an Ixodes tick. Int J Med Microbiol. 2006;296:157-61.

45. Stuen S, Granquist EG, Silaghi C. Anaplasma phagocytophilum-a widespread multi-host pathogen with highly adaptive strategies. Front Cell Infect Microbiol. 2013;3:31.

46. Hanincova K, Schafer SM, Etti S, Sewell HS, Taragelova V, Ziak D, et al. Association of Borrelia afzelii with rodents in Europe. Parasitology. 2003;126(Pt 1):11-20.

47. Burri C, Schumann O, Schumann C, Gern L. Are Apodemus spp. mice and Myodes glareolus reservoirs for Borrelia miyamotoi, Candidatus Neoehrlichia mikurensis, Rickettsia helvetica, R. monacensis and Anaplasma phagocytophilum? Ticks Tick Borne Dis. 2014;5(3):245-51.

48. Eisenhofer R, Minich JJ, Marotz C, Cooper A, Knight R, Weyrich LS. Contamination in low microbial biomass microbiome studies: issues and recommendations. Trends Microbiol. 2019;27(2):105-17.

49. Zhang XC, Yang ZN, Lu B, Ma XF, Zhang CX, Xu HJ. The composition and transmission of microbiome in hard tick, Ixodes persulcatus, during blood meal. Ticks Tick Borne Dis. 2014;5(6):864-70.

50. Tokarz R, Tagliafierro T, Sameroff S, Cucura DM, Oleynik A, Che X, et al. Microbiome analysis of Ixodes scapularis ticks from New York and Connecticut. Ticks Tick Borne Dis. 2019;10(4):894-900.

51. Vandeputte D, Kathagen G, D'hoe K, Vieira-Silva S, Valles-Colomer M, Sabino J, et al. Quantitative microbiome profiling links gut community variation to microbial load. Nature. 2017;551(7681):507-11. 
52. Duron O, Binetruy F, Noël V, Cremaschi J, McCoy KD, Arnathau C, et al. Evolutionary changes in symbiont community structure in ticks. Mol Ecol. 2017;26(11):2905-21.

53. Lowe CD, Minter EJ, Cameron DD, Brockhurst MA. Shining a light on exploitative host control in a photosynthetic endosymbiosis. Curr Biol. 2016;26(2):207-11.

54. Rio RV, Wu Y-n, Filardo G, Aksoy S. Dynamics of multiple symbiont density regulation during host development: tsetse fly and its microbial flora. Proc Royal Soc B Biol Sci. 2006;273(1588):805-14.

55. Duron O, Morel O, Noël V, Buysse M, Binetruy F, Lancelot R, et al. Tick-bacteria mutualism depends on B vitamin synthesis pathways. Curr Biol. 2018;28(12):1896-902. e5.

56. Oliver KM, Russell JA, Moran NA, Hunter MS. Facultative bacterial symbionts in aphids confer resistance to parasitic wasps. Proc Nati Acad Sci. 2003;100(4):1803-7.

57. Russell JA, Moran NA. Costs and benefits of symbiont infection in aphids: variation among symbionts and across temperatures. Proc Royal Soc B Biol Sci. 2006;273(1586):603-10.

58. Hunter DJ, Torkelson JL, Bodnar J, Mortazavi B, Laurent T, Deason J, et al. The Rickettsia endosymbiont of Ixodes pacificus contains all the genes of de novo folate biosynthesis. PloS one. 2015;10(12):e0144552.

59. Steiner FE, Pinger RR, Vann CN, Grindle N, Civitello D, Clay K, et al. Infection and co-infection rates of Anaplasma phagocytophilum variants, Babesia spp., Borrelia burgdorferi, and the rickettsial endosymbiont in Ixodes scapularis (Acari: Ixodidae) from sites in Indiana, Maine, Pennsylvania, and Wisconsin. J Med Entomol. 2014;45(2):289-97.

60. Tokarz R, Tagliafierro T, Sameroff S, Cucura DM, Oleynik A, Che X, et al. Microbiome analysis of Ixodes scapularis ticks from New York and Connecticut. Ticks Tick Borne Dis. 2019;10(4):894-900.

61. Ross BD, Hayes B, Radey MC, Lee X, Josek T, Bjork J, et al. Ixodes scapularis does not harbor a stable midgut microbiome. ISME J. 2018;12(11):2596-607.

62. Hammer TJ, Sanders JG, Fierer N. Not all animals need a microbiome. FEMS Microbiol Ecol. 2019;366(10):fnz117.

63. Narasimhan S, Swei A, Abouneameh S, Pal U, Pedra JH, Fikrig E. Grappling with the tick microbiome. Trends Parasitol. 2021.

64. Gofton AW, Oskam CL, Lo N, Beninati T, Wei H, McCarl V, et al. Inhibition of the endosymbiont "Candidatus Midichloria mitochondrii" during 16S rRNA gene profiling reveals potential pathogens in Ixodes ticks from Australia. Parasit Vectors. 2015;8(1):345.

65. Gundel PE, Rudgers J, Ghersa CM. Incorporating the process of vertical transmission into understanding of host-symbiont dynamics. Oikos. 2011;120(8):1121-8.

66. Oliver KM, Campos J, Moran NA, Hunter MS. Population dynamics of defensive symbionts in aphids. Proc Royal Soc B. 2008;275(1632):293-9.

\section{Figures}




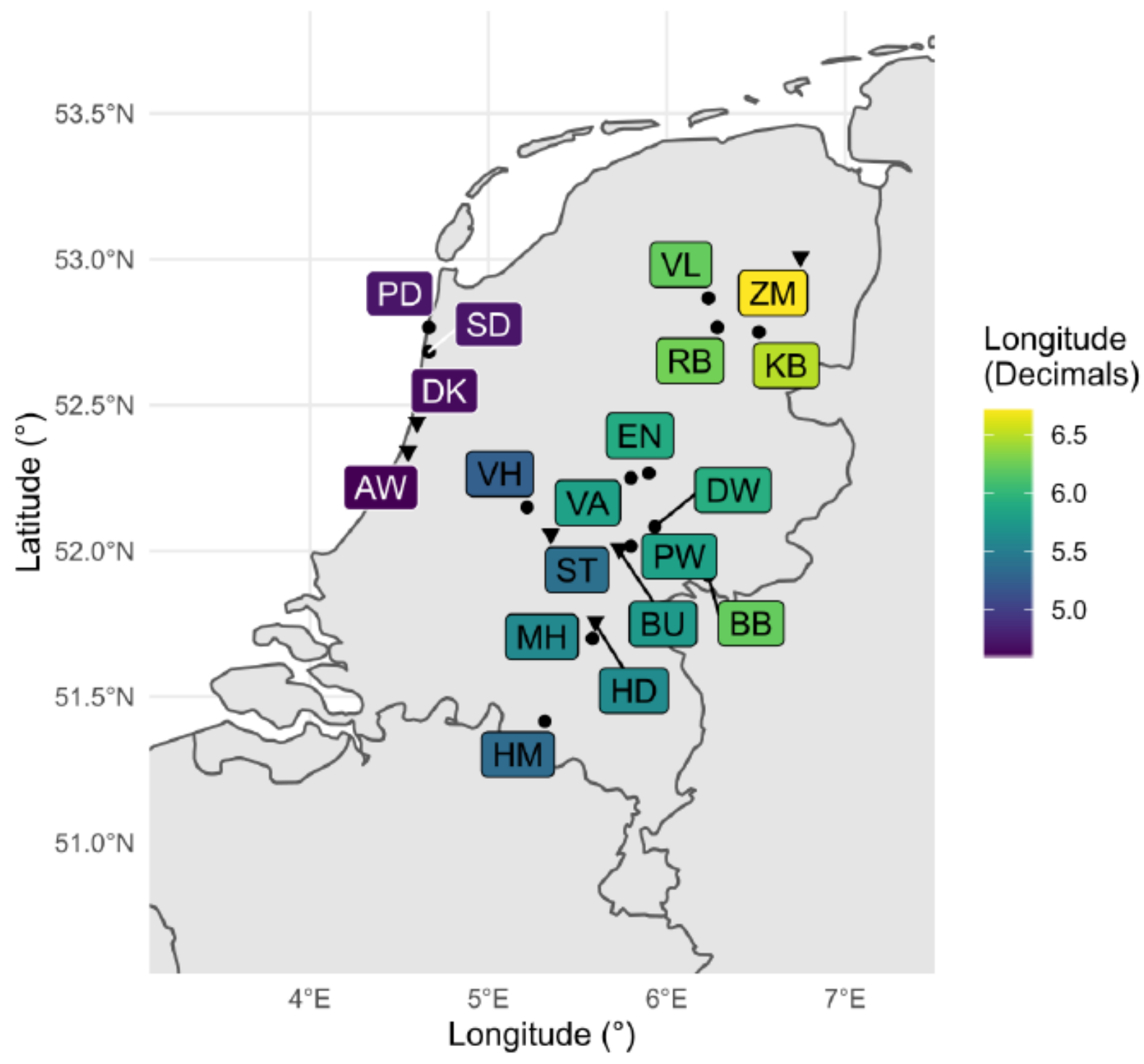

Figure 1

Sampling sites of I. ricinus in The Netherlands 


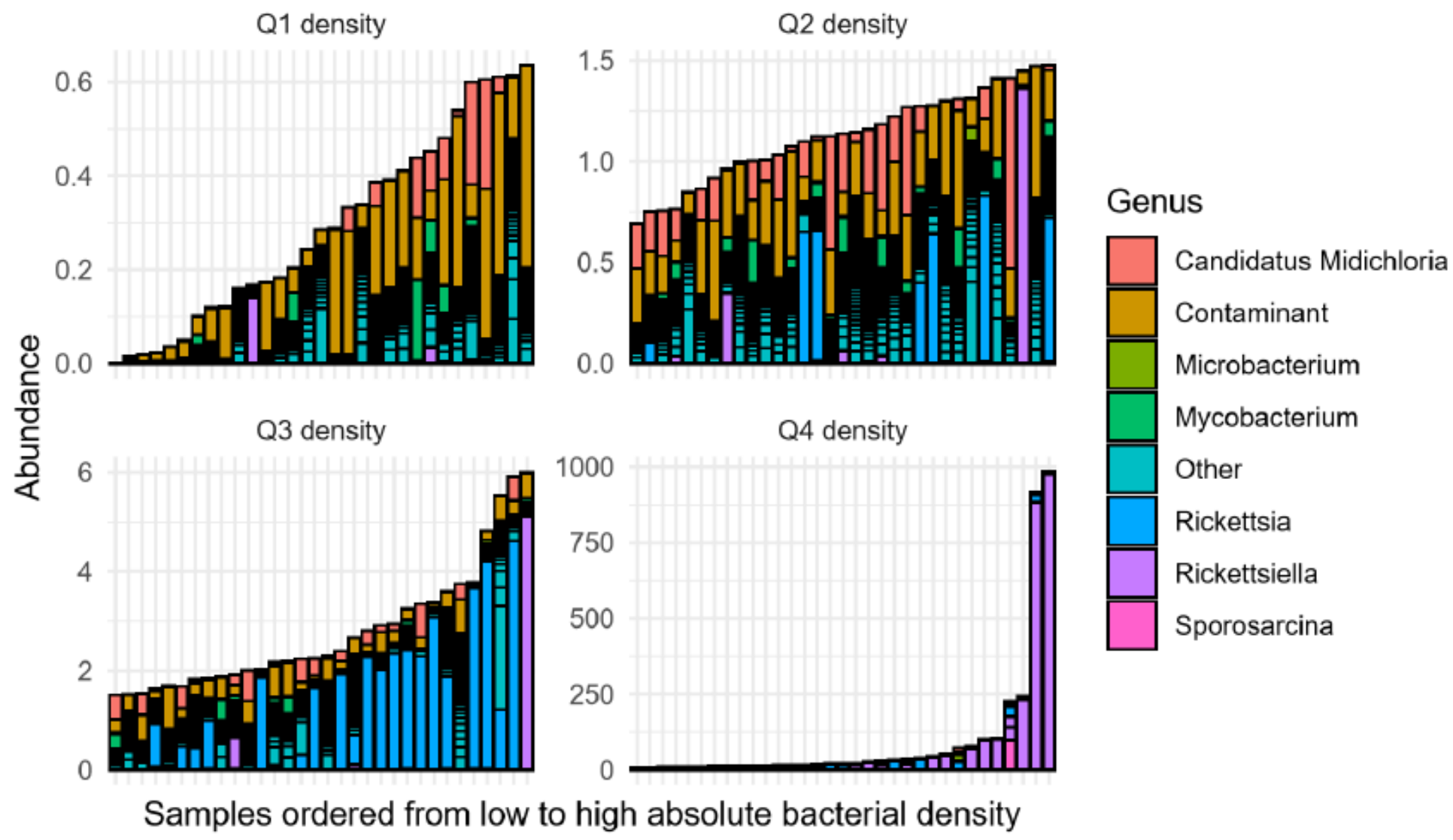

Figure 2

Abundances (number of reads; $Y$-axis) of the ten most abundant bacterial taxa in individual and pooled I. ricinus samples (X-axis). 

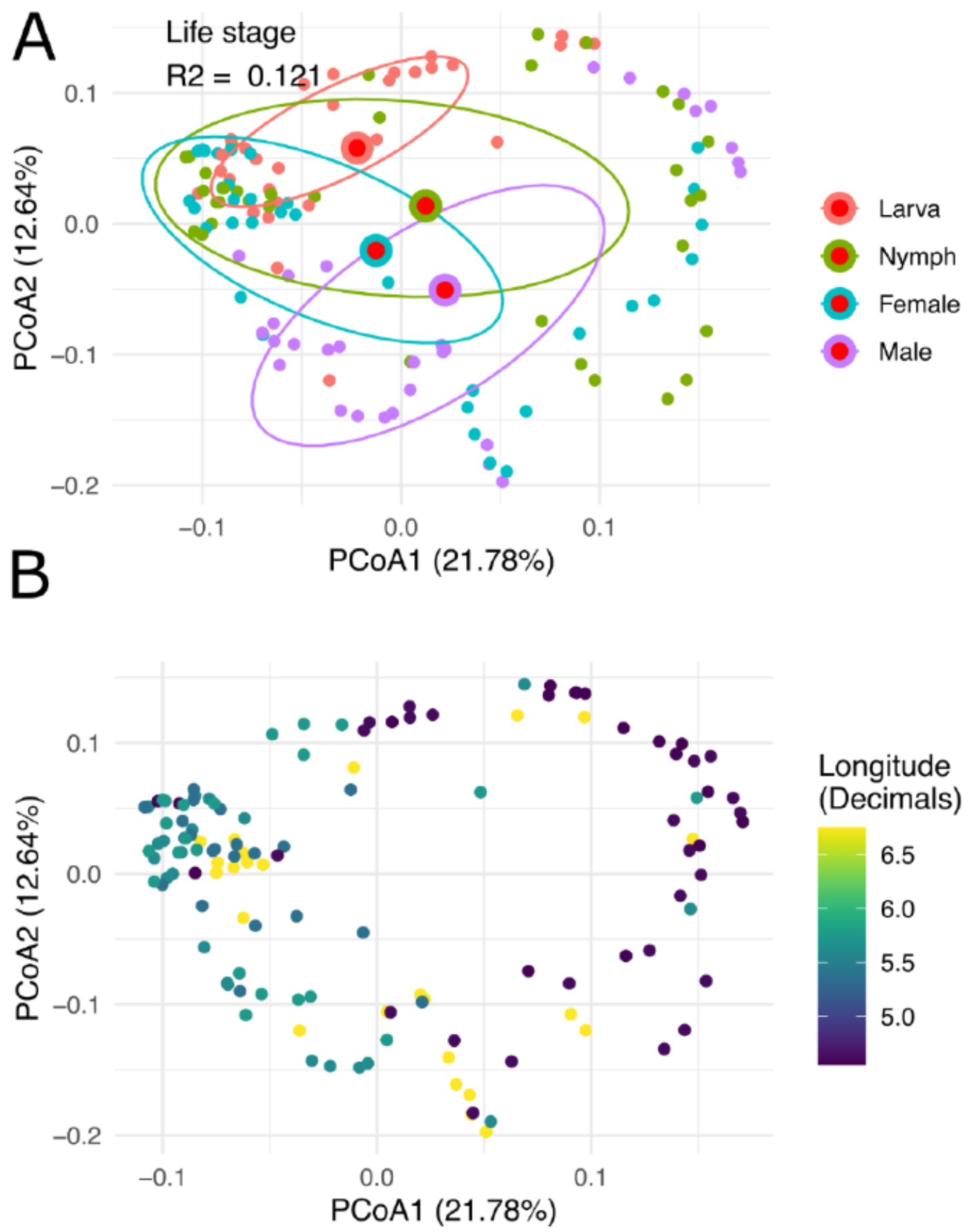

Figure 3

Bacterial community variation in Ixodes ricinus across life stages and longitude. (a) Principal Coordinate Analysis of Bray-Curtis dissimilarities overlaid with centroids of tick life stage. Data ellipses contain $50 \%$ of the samples belonging to the different life stages. (b) Principal Coordinate Analysis of Bray-Curtis dissimilarities, coloured by longitude. 


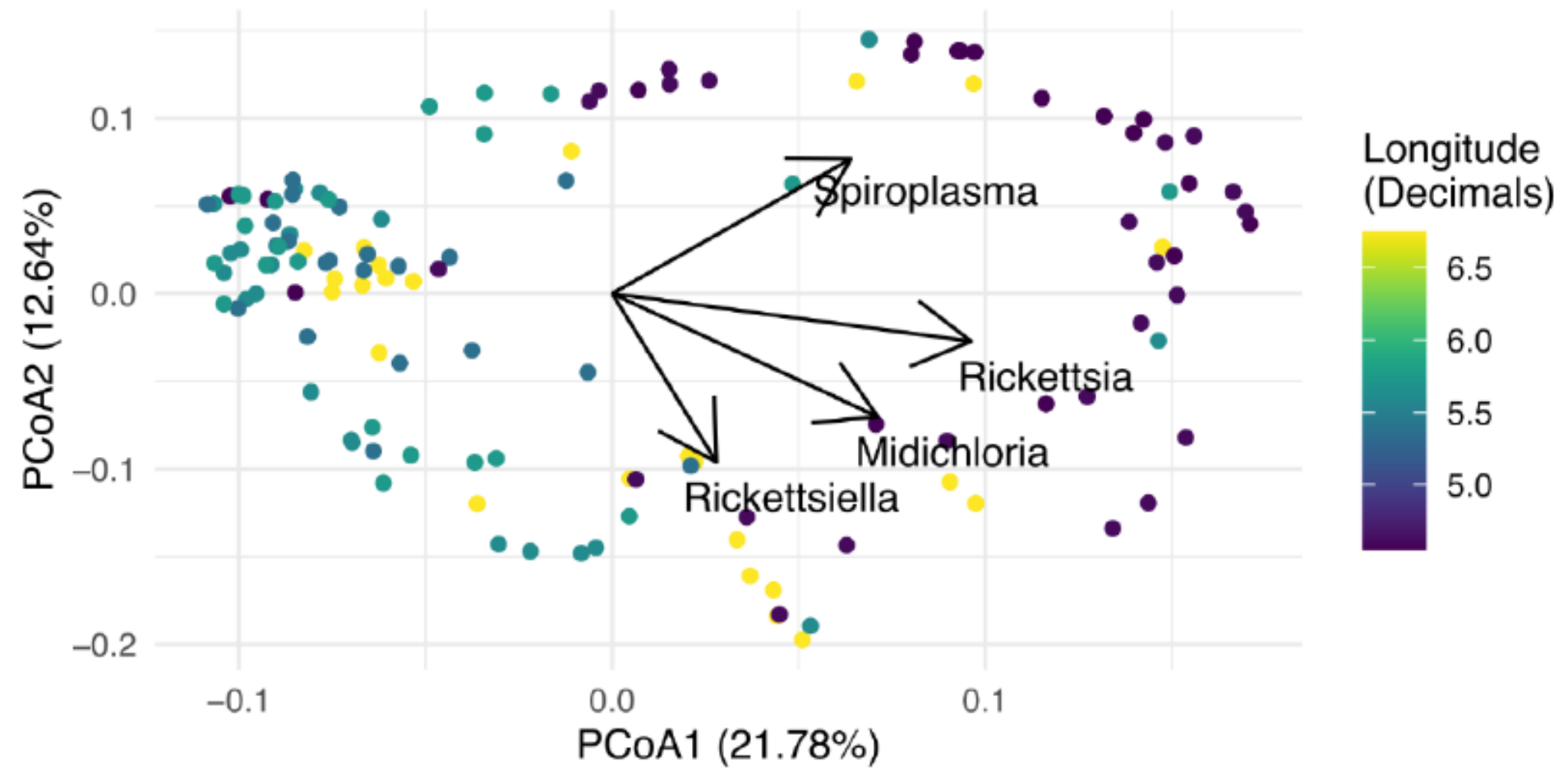

Figure 4

Rickettsia abundance covaries with community composition.

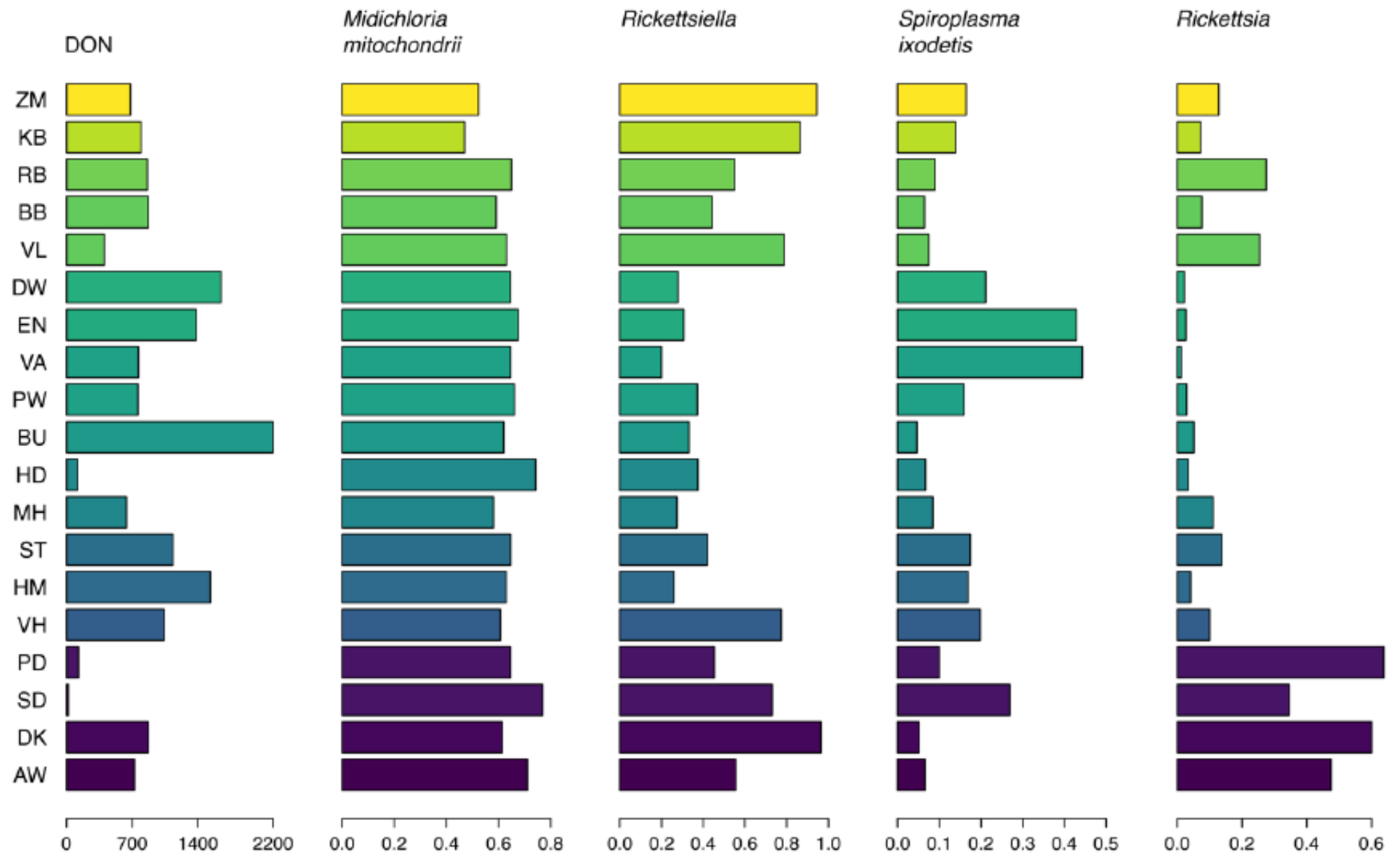




\section{Figure 5}

Local occurrence of vertically-transmitted microorganisms. The leftmost column shows the density of nymphs (DON) per forest site (two letters labels). The remaining columns show the prevalence of vertically-transmitted tick symbionts. Locations are coloured by their longitude.

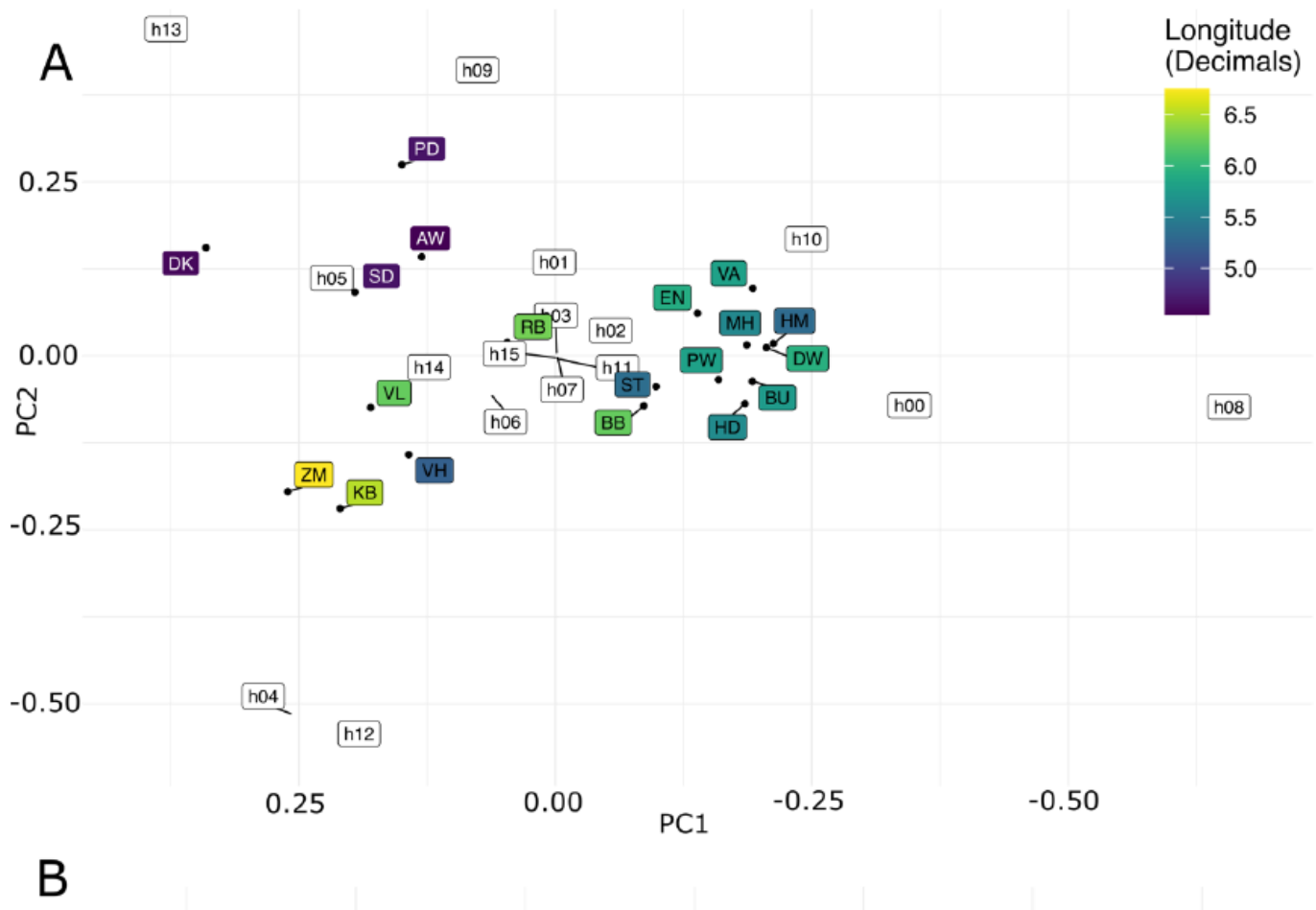

0.1

h04

hoo

บับ

$-0.1$
0.1
0.0
$-0.1$

h08

\section{Figure 6}

Relative occurrence of symbionts in I. ricinus nymphs from 19 forest sites coloured by their latitude. (a) Relative occurrence of vertically-transmitted symbionts such as R. helvetica, M. mitochondrii, S. ixodetis, and Rickettsiella spp. (b) Relative occurrence of horizontally-transmitted pathogens such as A. phagocytophilum, B. afzelii, B. garinii and B. valaisiana (combined), and N. mikurensis. Tick populations from the forest sites situated close to each other 
(see Figure 5) clearly share a similar composition of vertically-transmitted symbionts but not horizontallytransmitted pathogens. Transparent boxes show haplotype numbers, which correspond to symbiont combinations in individual ticks; for example, in panel (a), the forest sites from the centre of the Netherlands are dominated by nymphs infected only with M. mitochondrii (h08) or with none of the symbionts (h00). Please note the different scales of axes between the two figures. A full description of each haplotype is provided in Additional file 4: Table S9.

\section{Supplementary Files}

This is a list of supplementary files associated with this preprint. Click to download.

- Additionalfile1.xlsx

- Additionalfile2.docx

- Additionalfile3.xlsx

- Additionalfile4.xlsx

- additionalfileinfo.docx 